\title{
Simulation and modeling of synthesis $\mathrm{Cu}$ nanoparticles in sodium alginate media by means of expert systems
}

\begin{abstract}
This research was to apply the combination of the particle swarm optimization method and artificial neural network training with the aim of building a quantitative model to forecast the size of copper nanoparticles (Cu-NPs) prepared in sodium alginate. Sodium alginate, sodium hydroxide, copper sulfate, hydrazinium hydroxide, and ascorbic acid were used as stabilizer, $\mathrm{pH}$ moderator, copper precursor, reducing agent, and antioxidant, respectively. The results showed that the different sizes of $\mathrm{Cu}$-NPs were obtained by changing these functions. Meaning that by increasing the amount of sodium alginate and or increase the volume of hydrazine hydrate, particle sizes of $\mathrm{Cu}-\mathrm{NPs}$ were reduced. Other variables had the opposite effects due to the increase of the size of the $\mathrm{Cu}$-NPs. The prediction results were remarkably in agreement with the experimental data with a correlation coefficient of 0.99 and a mean square error of 0.0058 .
\end{abstract}

Keyword: Artificial neural network; Copper nanoparticles; Particle swarm optimization; Sodium alginate 